\title{
MULTIPLE POSITIVE SOLUTIONS FOR SINGULARLY PERTURBED ELLIPTIC PROBLEMS IN EXTERIOR DOMAINS
}

\author{
Giovanna CERAMI ${ }^{\mathrm{a}, *}$, Riccardo MOLLE ${ }^{\mathrm{b}}$ \\ ${ }^{a}$ Dipartimento di Matematica ed Applicazioni, Università di Palermo, Via Archirafi n. 34, \\ 90123 Palermo, Italy \\ b Dipartimento di Matematica, Università di Roma "Tor Vergata", Via della Ricerca Scientifica n. 1, \\ 00133 Roma, Italy
}

Received 3 March 2002, revised 20 June 2002

ABSTRACT. - The equation $-\varepsilon^{2} \Delta u+a_{\varepsilon}(x) u=u^{p-1}$ with boundary Dirichlet zero data is considered in an exterior domain $\Omega=\mathbb{R}^{N} \backslash \bar{\omega}(\omega$ bounded and $N \geqslant 2)$. Under the assumption that $a_{\varepsilon} \geqslant a_{0}>0$ concentrates round a point of $\Omega$ as $\varepsilon \rightarrow 0$, that $p>2$ and $p<2 N /(N-2)$ when $N \geqslant 3$, the existence of at least three positive distinct solutions is proved.

(c) 2003 L'Association Publications de l'Institut Henri Poincaré. Published by Elsevier B.V. All rights reserved

MSC: $35 \mathrm{~J} 20 ; 35 \mathrm{~J} 60$

Keywords: Exterior domains; Lack of compactness; Multiplicity of solutions

RÉSUMÉ. - Dans cet article on étude l'équation $-\varepsilon^{2} \Delta u+a_{\varepsilon}(x) u=u^{p-1}$ dans l'ouvert extérieur $\Omega=\mathbb{R}^{N} \backslash \bar{\omega}$ ( $\omega$ borné et $N \geqslant 2$ ), avec la condition de Dirichlet $u=0$ sur $\partial \Omega$. En supposant que $a_{\varepsilon} \geqslant a_{0}>0$ se concentre autour d'un point du domaine $\Omega$ quand $\varepsilon \rightarrow 0$, que $p>2$ et que $p<2 N /(N-2)$ quand $N \geqslant 3$, on démontre que le problème possède au moins trois solutions distinctes.

C 2003 L'Association Publications de l'Institut Henri Poincaré. Published by Elsevier B.V. All rights reserved

\section{Introduction}

In this paper we consider the problem

$$
\left(P_{\varepsilon}\right) \quad \begin{cases}-\varepsilon^{2} \Delta u+a_{\varepsilon}(x) u=u^{p-1} & \text { in } \Omega, \\ u>0 & \text { in } \Omega, \\ u=0 & \text { on } \partial \Omega,\end{cases}
$$

\footnotetext{
Work supported by Italian M.I.U.R., national research project "Metodi variazionali e topologici nello studio di fenomeni non lineari”.

* Corresponding author.

E-mail addresses: cerami@math.unipa.it (G. Cerami), molle@mat.uniroma2.it (R. Molle).
} 
where $\Omega=\mathbb{R}^{N} \backslash \bar{\omega}, \omega$ being a nonempty, bounded domain having smooth boundary $\partial \omega=\partial \Omega, N \geqslant 2, \varepsilon \in \mathbb{R}^{+} \backslash\{0\}, p>2$ and $p<2 N /(N-2)$ when $N \geqslant 3 . a_{\varepsilon}$ is a given nonnegative function that, as $\varepsilon \rightarrow 0$, concentrates round a point $x_{0} \in \Omega$, namely $a_{\varepsilon}$ has the form

$$
a_{\varepsilon}(x)=a_{0}+\alpha\left(\frac{x-x_{0}}{\varepsilon}\right)
$$

and satisfies

$$
\begin{aligned}
& \left(A_{1}\right) \quad a_{0} \in \mathbb{R}^{+} \backslash\{0\}, x_{0} \in \Omega, \alpha(x) \geqslant 0, \alpha \in L^{N / 2}\left(\mathbb{R}^{N}\right),|\alpha|_{L^{N / 2}\left(\mathbb{R}^{N}\right)} \neq 0, \\
& \left(A_{2}\right) \int_{\mathbb{R}^{N}} \alpha(x) \mathrm{e}^{2|x|}\left(1+|x|^{\frac{N-1}{2} \sigma}\right) d x<\infty \text { for some } \sigma \in(1,2] .
\end{aligned}
$$

Problem $\left(P_{\varepsilon}\right)$ has a variational structure: the solutions of $\left(P_{\varepsilon}\right)$ can be characterized as the nonnegative functions that are critical points of the functional $\mathcal{I}_{\varepsilon}: H_{0}^{1}(\Omega) \rightarrow \mathbb{R}$

$$
\mathcal{I}_{\varepsilon}(u)=\int_{\Omega}\left(\varepsilon^{2}|\nabla u|^{2}+a_{\varepsilon}(x) u^{2}\right) d x
$$

constrained to lie on the manifold

$$
\mathcal{M}=\left\{\left.u \in H_{0}^{1}(\Omega)|| u\right|_{L^{p}(\Omega)}=1\right\} .
$$

However, it is well known that the unboundedness of the domain gives rise to a lack of compactness, not allowing a straight application of the usual variational techniques. In particular $\left(P_{\varepsilon}\right)$ cannot be solved by minimization, in fact (see Section 2), the infimum of $\mathcal{I}_{\varepsilon}$ on $\mathcal{M}$ is not achieved, moreover the functional $\mathcal{I}_{\varepsilon}$ does not satisfy the Palais-Smale condition in every energy level (see [1] and [3] for a careful analysis of the compactness question). The study of $\left(P_{\varepsilon}\right)$ needs subtle tools as the minimax theory together with topological arguments.

In recent years problems like $\left(P_{\varepsilon}\right)$ have been object of several researches, here we only recall that, without any symmetry assumption on $\omega$, the existence of one solution for $\left(P_{\varepsilon}\right)$ has been proved, first, in [3], in the case $a_{\varepsilon}(x) \equiv a_{0}$, then in [1], under more general assumptions; multiplicity results have been obtained, when $a_{\varepsilon}(x) \equiv a_{0}$, in domains having several holes $[7,8,11,15]$ relating the number of solutions of $\left(P_{\varepsilon}\right)$ to the metric and/or topological properties of $\Omega$. We also remark that, for equations in $\mathbb{R}^{N}$ having nonconstant, nonsymmetric coefficients, the existence of one positive solution has been stated in [2,4], while multiple solutions have been found in [13].

In this work, motivated by former results, [6,9], that emphasize the role that a concentrating potential $a_{\varepsilon}$ can play in obtaining multiplicity of solutions for problems like $\left(P_{\varepsilon}\right)$ in bounded domains, we investigate the effect of such a potential when $\Omega$ is an unbounded exterior domain.

The result we obtain is stated in the following

THEOREM 1.1. - Let $a_{\varepsilon}$ be as in (1.1) and let the assumptions $\left(A_{1}\right)$ and $\left(A_{2}\right)$ be satisfied. Then there exists $\bar{\varepsilon}>0$ such that for every $\varepsilon \in(0, \bar{\varepsilon})$ Problem $\left(P_{\varepsilon}\right)$ has at least three distinct solutions $u_{1, \varepsilon}, u_{2, \varepsilon}, u_{3, \varepsilon}$. Moreover 


$$
\begin{aligned}
& \lim _{\varepsilon \rightarrow 0} \frac{1}{\varepsilon^{N(1-2 / p)}} \mathcal{I}_{\varepsilon}\left(\frac{u_{1, \varepsilon}}{\left|u_{1, \varepsilon}\right|_{L^{p}(\Omega)}}\right)=m, \\
& \lim _{\varepsilon \rightarrow 0} \frac{1}{\varepsilon^{N(1-2 / p)}} \mathcal{I}_{\varepsilon}\left(\frac{u_{2, \varepsilon}}{\left|u_{2, \varepsilon}\right|_{L^{p}(\Omega)}}\right) \in\left(m, 2^{1-2 / p} m\right), \\
& \lim _{\varepsilon \rightarrow 0} \frac{1}{\varepsilon^{N(1-2 / p)}} \mathcal{I}_{\varepsilon}\left(\frac{u_{3, \varepsilon}}{\left|u_{3, \varepsilon}\right|_{L^{p}(\Omega)}}\right)=2^{1-2 / p} m,
\end{aligned}
$$

where

$$
m=\inf \left\{\left.\int_{\mathbb{R}^{N}}\left[|\nabla u|^{2}+a_{0} u^{2}\right] d x\left|u \in H^{1}\left(\mathbb{R}^{N}\right),\right| u\right|_{L^{p}\left(\mathbb{R}^{N}\right)}=1\right\} .
$$

We remark that the above theorem gives the existence of at least three solutions whatever $\Omega$ is, even the complement of a convex domain.

It is worth observing, also, that the asymptotic energy estimates give some information about the shape of the solutions. Indeed $u_{1, \varepsilon}$ is a "single peak" solution, that is a function that, suitably translated and scaled, tends, as $\varepsilon \rightarrow 0$, to a solution of the limit problem

$$
\left(P_{\infty}\right) \begin{cases}-\Delta u+a_{0} u=u^{p-1} & \text { in } \mathbb{R}^{N}, \\ u>0 & \text { in } \mathbb{R}^{N}, \\ u(x) \rightarrow 0 & \text { as }|x| \rightarrow \infty\end{cases}
$$

and, on the other hand, $u_{3, \varepsilon}$ must be a "two-peaks" solution, in fact its energy, suitably scaled, tends to the energy of a pairs of not interacting solutions of $\left(P_{\infty}\right)$. About the last solution, $u_{2, \varepsilon}$, we can guess (but we have not a rigorous proof) that it, suitably scaled in $x_{0}$, as $\varepsilon \rightarrow 0$, tends to a solution of

$$
\left(P_{\alpha}\right) \begin{cases}-\Delta u+\left(a_{0}+\alpha(x)\right) u=u^{p-1} & \text { in } \mathbb{R}^{N}, \\ u>0 & \text { in } \mathbb{R}^{N}, \\ u(x) \rightarrow 0 & \text { as }|x| \rightarrow \infty\end{cases}
$$

whose shape depends on $\alpha$ (see [13]).

Finally, we point out that we can look at problem $\left(P_{\varepsilon}\right)$ in a "dual" way: an equation not depending on $\varepsilon$, considered in an exterior domain whose complement, as $\varepsilon \rightarrow 0$, widens and becomes far and far from the relevant part (in the sense of $L^{N / 2}\left(\mathbb{R}^{N}\right)$ ) of $\alpha$.

Actually, considering, for instance $\Omega_{\varepsilon, x_{0}}=\left\{x \in \mathbb{R}^{N} \mid \varepsilon x+x_{0} \in \Omega\right\}$ an easy scale change shows that to any solution of $\left(P_{\varepsilon}\right)$ there corresponds, in a one to one way, a solution of

$$
\begin{cases}-\Delta u+\left(a_{0}+\alpha(x)\right) u=u^{p-1} & \text { in } \Omega_{\varepsilon, x_{0}}, \\ u>0 & \text { in } \Omega_{\varepsilon, x_{0}} \\ u=0 & \text { on } \partial \Omega_{\varepsilon, x_{0}} .\end{cases}
$$

Thus the conclusion of Theorem 1.1 can be expressed equivalently as follows:

THEOREM 1.2. - Let $a_{0}$ and $\alpha$ satisfy $\left(A_{1}\right)$ and $\left(A_{2}\right)$. Let $\Omega_{n} \subset \mathbb{R}^{N}$ be a sequence of exterior domains such that for some $y_{n} \in \mathbb{R}^{N}$ and $r_{n} \rightarrow \infty$

$$
B\left(y_{n}, r_{n}\right) \subset \mathbb{R}^{N} \backslash \Omega_{n}, \quad B\left(x_{0}, r_{n}\right) \subset \Omega_{n} .
$$


Then there exists $\bar{n} \in \mathbb{N}$ such that for all $n>\bar{n}$ the equation $-\Delta u+\left(a_{0}+\alpha(x)\right) u=$ $u^{p-1}$ with zero Dirichlet boundary data in $\Omega_{n}$ has at least three positive solutions, $\bar{u}_{1, n}$, $\bar{u}_{2, n}, \bar{u}_{3, n}$. Moreover

$$
\begin{gathered}
\lim _{n \rightarrow+\infty} \frac{\int_{\Omega_{n}}\left(\left|\nabla \bar{u}_{1, n}(x)\right|^{2}+\left(a_{0}+\alpha(x)\right) \bar{u}_{1, n}^{2}(x)\right) d x}{\left|\bar{u}_{1, n}\right|_{L^{p}\left(\Omega_{n}\right)}^{2}}=m, \\
\lim _{n \rightarrow+\infty} \frac{\int_{\Omega_{n}}\left(\left|\nabla \bar{u}_{2, n}(x)\right|^{2}+\left(a_{0}+\alpha(x)\right) \bar{u}_{2, n}^{2}(x)\right) d x}{\left|\bar{u}_{2, n}\right|_{L^{p}\left(\Omega_{n}\right)}^{2}} \in\left(m, 2^{1-2 / p} m\right), \\
\lim _{n \rightarrow+\infty} \frac{\int_{\Omega_{n}}\left(\left|\nabla \bar{u}_{3, n}(x)\right|^{2}+\left(a_{0}+\alpha(x)\right) \bar{u}_{3, n}^{2}(x)\right) d x}{\left|\bar{u}_{3, n}\right|_{L^{p}\left(\Omega_{n}\right)}^{2}}=2^{1-2 / p} m .
\end{gathered}
$$

The paper is organized as follows: Section 2 is devoted to introducing some notations and recalling some known results and useful relations; in Section 3 some useful tools are introduced and some basic asymptotic estimates are proved, Section 4 contains the proof of Theorem 1.1. Arguing as in proving Theorem 1.1, it is a simple matter to get the proof of Theorem 1.2.

\section{Notations, known facts and useful remarks}

Throughout the paper we make use of the following notations.

- $L^{p}(\mathcal{D}), 1 \leqslant p<+\infty, \mathcal{D} \subseteq \mathbb{R}^{N}$, denotes a Lebesgue space; the norm in $L^{p}(\mathcal{D})$ is denoted by $|\cdot|_{p, \mathcal{D}}$.

- $H_{0}^{1}(\mathcal{D}), \mathcal{D} \subset \mathbb{R}^{N}$ and $H^{1}\left(\mathbb{R}^{N}\right)$ denote the Sobolev spaces obtained, respectively, as closure of $C_{0}^{\infty}(\mathcal{D})$ and $C_{0}^{\infty}\left(\mathbb{R}^{N}\right)$ with respect to the norms

$$
\|u\|_{\mathcal{D}}=\left[\int_{\mathcal{D}}\left(|\nabla u|^{2}+a_{0} u^{2}\right) d x\right]^{1 / 2}, \quad\|u\|_{\mathbb{R}^{N}}=\left[\int_{\mathbb{R}^{N}}\left(|\nabla u|^{2}+a_{0} u^{2}\right) d x\right]^{1 / 2} .
$$

- If $\mathcal{D}_{1} \subset \mathcal{D}_{2} \subseteq \mathbb{R}^{N}$ and $u \in H_{0}^{1}\left(\mathcal{D}_{1}\right)$, we denote also by $u$ its extension to $\mathcal{D}_{2}$ obtained setting $u \equiv 0$ outside $\mathcal{D}_{1}$.

- $\mathcal{D}_{\varepsilon}$ denotes the subset of $\mathbb{R}^{N}\left\{y \in \mathbb{R}^{N} \mid \varepsilon y \in \mathcal{D}\right\}, \mathcal{D} \subset \mathbb{R}^{N}$.

- $B(y, \rho)$ denotes the open ball, of $\mathbb{R}^{N}$, having radius $\rho$ and centered at $y$.

In what follows, without any loss of generality, we assume $a_{0}=1$ and $x_{0}=0$.

Setting

$$
u_{\varepsilon}(x)=\varepsilon^{N / p} u(\varepsilon x)
$$

an easy computation shows that for every $u \in H_{0}^{1}(\Omega) u_{\varepsilon} \in H_{0}^{1}\left(\Omega_{\varepsilon}\right), u \in \mathcal{M}$ if and only if $\left|u_{\varepsilon}\right|_{p, \Omega_{\varepsilon}}=1$ and

$$
\begin{aligned}
\mathcal{I}_{\varepsilon}(u) & =\int_{\Omega}\left[\varepsilon^{2}|\nabla u|^{2}+\left(1+\alpha\left(\frac{x}{\varepsilon}\right)\right) u^{2}\right] d x \\
& =\varepsilon^{(1-2 / p) N} \int_{\Omega_{\varepsilon}}\left[\left|\nabla u_{\varepsilon}\right|^{2}+(1+\alpha(x)) u_{\varepsilon}^{2}\right] d x .
\end{aligned}
$$


Thus looking for critical points of $\mathcal{I}_{\varepsilon}$ on $\mathcal{M}$ is equivalent to searching for critical points of the "rescaled" energy functional

$$
E_{\varepsilon}(u)=\int_{\Omega_{\varepsilon}}\left[|\nabla u|^{2}+(1+\alpha(x)) u^{2}\right] d x
$$

on the manifold

$$
M_{\varepsilon}=\left\{\left.u \in H_{0}^{1}\left(\Omega_{\varepsilon}\right)|| u\right|_{p, \Omega_{\varepsilon}}=1\right\} .
$$

Let us set

$$
m_{\varepsilon}=\inf \left\{E_{\varepsilon}(u) \mid u \in M_{\varepsilon}\right\}
$$

and

$$
m=\inf \left\{\left.\|u\|_{\mathbb{R}^{N}}^{2}\left|u \in H^{1}\left(\mathbb{R}^{N}\right),\right| u\right|_{p, \mathbb{R}^{N}}=1\right\} .
$$

The infimum in (2.3) is achieved (see [16] or [5]) by a positive function $w$, that is unique modulo translations (see [12]) and radially symmetric about the origin, decreasing when the radial co-ordinate increases and such that

$$
\lim _{|x| \rightarrow+\infty}\left|D^{j} w(x)\right||x|^{\frac{N-1}{2}} \mathrm{e}^{|x|}=d_{j}>0, \quad d_{j} \in \mathbb{R}, j=0,1
$$

(see [5] and [10]).

On the contrary we have

PROPOSITION 2.1. - Let $\alpha$ satisfy $\left(A_{1}\right)$. Then

$$
m_{\varepsilon}=m
$$

and the minimization problem (2.2) has no solution.

Proof. - Since we may consider $H_{0}^{1}\left(\Omega_{\varepsilon}\right)$ as a subspace of $H^{1}\left(\mathbb{R}^{N}\right)$,

$$
m_{\varepsilon} \geqslant m
$$

To prove that the equality holds, we consider the sequence

$$
w_{\varepsilon, y_{n}}(x):=\frac{\phi_{\varepsilon}(x) w\left(x-y_{n}\right)}{\left|\phi_{\varepsilon}(x) w\left(x-y_{n}\right)\right|_{p, \Omega_{\varepsilon}}}
$$

where $y_{n} \in \Omega_{\varepsilon}, \lim _{n \rightarrow+\infty}\left|y_{n}\right|=+\infty, w$ is the function realizing (2.3) and $\phi_{\varepsilon}(x)=$ $\phi(\varepsilon x)$ with $\phi: \mathbb{R}^{N} \rightarrow[0,1]$ a $C^{\infty}$-function such that: $\phi(x)=0$ if $x \in \omega, 0 \leqslant \phi(x) \leqslant 1$, $\operatorname{supp}(1-\phi)$ is compact, and we show that

$$
\lim _{n \rightarrow+\infty} E_{\varepsilon}\left(w_{\varepsilon, y_{n}}\right)=m
$$

Indeed, using (2.4) it is not difficult to show that 


$$
\begin{aligned}
& \left|\phi_{\varepsilon}(x) w\left(x-y_{n}\right)-w\left(x-y_{n}\right)\right|_{p, \mathbb{R}^{N}}=\mathrm{o}\left(1 /\left|y_{n}\right|\right), \\
& \left\|\phi_{\varepsilon}(x) w\left(x-y_{n}\right)-w\left(x-y_{n}\right)\right\|_{\mathbb{R}^{N}}=\mathrm{o}\left(1 /\left|y_{n}\right|\right) .
\end{aligned}
$$

On the other hand, for every fixed $\eta>0$, we can find $\rho=\rho(\eta)>0$ so that

$$
\left|\phi_{\varepsilon}(x) w\left(x-y_{n}\right)\right|_{\frac{2 N}{N-2}, \Omega_{\varepsilon} \backslash B\left(y_{n}, \rho\right)}<\eta
$$

and

$$
|\alpha|_{N / 2, B\left(y_{n}, \rho\right)}<\eta
$$

if $n$ is large enough; hence

$$
\begin{aligned}
& \int_{\Omega_{\varepsilon}} \alpha(x)\left[\phi_{\varepsilon}(x) w\left(x-y_{n}\right)\right]^{2} d x \\
& \quad=\int_{B\left(y_{n}, \rho\right)} \alpha(x)\left[\phi_{\varepsilon}(x) w\left(x-y_{n}\right)\right]^{2} d x+\int_{\Omega_{\varepsilon} \backslash B\left(y_{n}, \rho\right)} \alpha(x)\left[\phi_{\varepsilon}(x) w\left(x-y_{n}\right)\right]^{2} d x \\
& \quad \leqslant \eta\left|\phi_{\varepsilon}(x) w\left(x-y_{n}\right)\right|_{\frac{2 N}{N-2}, \mathbb{R}^{N}}+\eta|\alpha|_{N / 2, \mathbb{R}^{N}}
\end{aligned}
$$

from which

$$
\lim _{n \rightarrow+\infty} \int_{\Omega_{\varepsilon}} \alpha(x)\left[\phi_{\varepsilon}(x) w\left(x-y_{n}\right)\right]^{2} d x=0
$$

follows.

Hence (2.8), (2.9) and (2.10) give (2.7).

Let us now assume that the minimization problem (2.2) has a solution $u^{*} \geqslant 0$. Then

$$
m \leqslant\left\|u^{*}\right\|_{\mathbb{R}^{N}}^{2}=\left\|u^{*}\right\|_{\Omega_{\varepsilon}}^{2} \leqslant\left\|u^{*}\right\|_{\Omega_{\varepsilon}}^{2}+\int_{\Omega_{\varepsilon}} \alpha(x)\left(u^{*}(x)\right)^{2} d x=m .
$$

Thus we deduce

$$
u^{*}(x)=w\left(x-y^{*}\right) \quad \text { for some } y^{*} \in \mathbb{R}^{N}
$$

and, by $\left(A_{1}\right)$ and $w(x)>0 \forall x \in \mathbb{R}^{N}$,

$$
0=\int_{\Omega_{\varepsilon}} \alpha(x)\left(u^{*}(x)\right)^{2} d x=\int_{\Omega_{\varepsilon}} \alpha(x) w^{2}\left(x-y^{*}\right) d x>0,
$$

a contradiction.

The functional $E_{\varepsilon}$ constrained on $M_{\varepsilon}$ does not verify globally the Palais-Smale condition, however, as proved in [3], the compactness is preserved in some energy range.

LEMMA 2.2. - Let $\left(u_{n}\right)_{n}$ be a Palais-Smale sequence for $E_{\varepsilon}$ constrained on $M_{\varepsilon}$, i.e. $u_{n} \in M_{\varepsilon}$

$$
\left\{\begin{array}{l}
\lim _{n \rightarrow \infty} E_{\varepsilon}\left(u_{n}\right)=c, \\
\lim _{n \rightarrow \infty} \nabla E_{\varepsilon \mid M_{\varepsilon}}\left(u_{n}\right)=0 .
\end{array}\right.
$$


If $c \in\left(m, 2^{1-2 / p} m\right)$ then $\left(u_{n}\right)_{n}$ is relatively compact.

The following lemma states a lower bound for the energy of a critical point $u$ of $E_{\varepsilon}$ on $M_{\varepsilon}$ that changes sign; the proof, that can be easily deduced using the definition of $m$, can be found in [7].

LEMMA 2.3. - Let $u \in H_{0}^{1}\left(\Omega_{\varepsilon}\right)$ be such that

$$
|u|_{p, \Omega_{\varepsilon}}=1, \quad E_{\varepsilon}(u)=c, \quad \nabla E_{\varepsilon \mid M_{\varepsilon}}(u)=0 .
$$

Then $u^{+} \not \equiv 0$ and $u^{-} \not \equiv 0$ implies $c>2^{1-2 / p} m$.

This lemma and the maximum principle ensure that critical points of $E_{\varepsilon}$ on $M_{\varepsilon}$ in the range $\left(m, 2^{1-2 / p} m\right)$ give rise to positive solutions of problem $\left(P_{\varepsilon}\right)$.

\section{Tools, preliminary remarks, basic estimates}

For what follows we need to introduce some barycenter type function.

For $u \in L^{p}\left(\mathbb{R}^{N}\right)$ we set

$$
\tilde{u}(x)=\frac{1}{|B(x, 1)|} \int_{B(x, 1)}|u(y)| d y
$$

$|B(x, 1)|$ being the Lebesgue measure of $B(x, 1)$, and

$$
\hat{u}(x)=\left[\tilde{u}(x)-\frac{1}{2} \max _{\mathbb{R}^{N}} \tilde{u}(x)\right]^{+} ;
$$

we then define $\beta: L^{p}\left(\mathbb{R}^{N}\right) \backslash\{0\} \rightarrow \mathbb{R}^{N}$ by

$$
\beta(u)=\frac{1}{|\hat{u}|_{p, \mathbb{R}^{N}}^{p}} \int_{\mathbb{R}^{N}} x[\hat{u}(x)]^{p} d x .
$$

We remark that $\beta$ is well defined for all $u \in L^{p}\left(\mathbb{R}^{N}\right) \backslash\{0\}$, because $\hat{u} \not \equiv 0$ and has compact support, moreover $\beta$ is continuous.

We define also, for every $\varepsilon>0$, another map $\beta_{\varepsilon}: L^{p}\left(\mathbb{R}^{N}\right) \backslash\{0\} \rightarrow \mathbb{R}^{N}$ by

$$
\beta_{\varepsilon}(u)=\frac{1}{|u|_{p, \mathbb{R}^{N}}^{p}} \int_{\mathbb{R}^{N}} \chi\left(x-\bar{x}_{\varepsilon}\right)|u(x)|^{p} d x
$$

where $\bar{x}_{\varepsilon}=\bar{x} / \varepsilon, \bar{x}$ being a fixed point in $\omega=\mathbb{R}^{N} \backslash \bar{\Omega}$ and $\chi$ is the function

$$
\chi(x)=\frac{x}{1+|x|} .
$$

We remark that $\beta_{\varepsilon}$ is a continuous map in $L^{p}\left(\mathbb{R}^{N}\right) \backslash\{0\}$; we observe also that $\beta_{\varepsilon}(w(x-$ $\left.\left.\bar{x}_{\varepsilon}\right)\right)=0$. 
We put

$$
\begin{aligned}
\mathcal{B}_{0}:=\inf \left\{\int_{\mathbb{R}^{N}}\left[|\nabla u|^{2}+(1+\alpha(x)) u^{2}\right] d x \mid\right. & u \in H^{1}\left(\mathbb{R}^{N}\right), \\
& \left.|u|_{p, \mathbb{R}^{N}}=1, \beta(u)=0\right\}
\end{aligned}
$$

and, for all $\varepsilon>0$, we set

$$
\begin{aligned}
& \mathcal{B}_{0, \varepsilon}:=\inf \left\{E_{\varepsilon}(u) \mid u \in M_{\varepsilon}, \beta(u)=0\right\}, \\
& \mathcal{B}_{\bar{x}_{\varepsilon}}:=\inf \left\{E_{\varepsilon}(u) \mid u \in M_{\varepsilon}, \beta(u)=\bar{x}_{\varepsilon}\right\}, \\
& \mathcal{B}_{0, \beta_{\varepsilon}}:=\inf \left\{E_{\varepsilon}(u) \mid u \in M_{\varepsilon}, \beta_{\varepsilon}(u)=0\right\} .
\end{aligned}
$$

We denote by $L_{\varepsilon}$ the segment joining 0 and $\bar{x}_{\varepsilon}$, i.e.

$$
L_{\varepsilon}=\left\{t \bar{x}_{\varepsilon} \mid t \in[0,1]\right\}
$$

and by

$$
\mathcal{A}_{\varepsilon}:=\inf \left\{E_{\varepsilon}(u) \mid u \in M_{\varepsilon}, \beta(u) \in L_{\varepsilon}\right\} .
$$

Fixed a point $\zeta \in \partial B(0,1)$ we denote by $\Sigma=\partial B(\zeta$, 2) i.e.

$$
\Sigma=\left\{z \in \mathbb{R}^{N}|| z-\zeta \mid=2\right\} .
$$

For every $\varepsilon>0$ and $\rho>0$ we define the operator

$$
\psi_{\varepsilon, \rho}: \Sigma \times[0,1] \rightarrow M_{\varepsilon}
$$

by

$$
\psi_{\varepsilon, \rho}[z, t](x)=\frac{\phi_{\varepsilon}(x)[(1-t) w(x-\rho z)+t w(x-\rho \zeta)]}{\left|\phi_{\varepsilon}(x)[(1-t) w(x-\rho z)+t w(x-\rho \zeta)]\right|_{p, \Omega_{\varepsilon}}}
$$

where $\phi_{\varepsilon}$ is the cut-off function introduced in Proposition 2.1 to define the sequence (2.6).

We put for all $z \in \mathbb{R}^{N}$

$$
w_{\varepsilon, z}(x)=\frac{\phi_{\varepsilon}(x) w(x-z)}{\left|\phi_{\varepsilon}(x) w(x-z)\right|_{p, \Omega_{\varepsilon}}}
$$

and we remark that $\forall z \in \Sigma$

$$
\psi_{\varepsilon, \rho}[z, 0](x)=w_{\varepsilon, \rho z}(x), \quad \psi_{\varepsilon, \rho}[z, 1](x)=w_{\varepsilon, \rho \zeta}(x) .
$$

We consider, also, for every $\rho>0$, the operator

$$
\psi_{\rho}: \Sigma \times[0,1] \rightarrow\left\{\left.u \in H^{1}\left(\mathbb{R}^{N}\right)|| u\right|_{p, \mathbb{R}^{N}}=1\right\}
$$


defined by

$$
\psi_{\rho}[z, t](x)=\frac{(1-t) w(x-\rho z)+t w(x-\rho \zeta)}{|(1-t) w(x-\rho z)+t w(x-\rho \zeta)|_{p, \mathbb{R}^{N}}} .
$$

Proposition 3.1. - Let $\alpha$ satisfy $\left(A_{1}\right)$. Let $\mathcal{B}_{0}, \mathcal{B}_{0, \varepsilon}$ and $m$ as defined, respectively, in (3.3), (3.4), (2.3). Then the relation

$$
\mathcal{B}_{0, \varepsilon} \geqslant \mathcal{B}_{0}>m
$$

holds for all $\varepsilon>0$.

Proof. - Clearly, $\forall \varepsilon>0, \mathcal{B}_{0, \varepsilon} \geqslant \mathcal{B}_{0}$ and $\mathcal{B}_{0} \geqslant m$, so, in order to prove (3.12), we have to show that the equality $\mathcal{B}_{0}=m$ cannot be true.

Arguing by contradiction, we assume $\mathcal{B}_{0}=m$. Hence a sequence of nonnegative functions $\left(u_{n}\right)_{n}$ in $H^{1}\left(\mathbb{R}^{N}\right)$ must exist so that

$$
\left.\begin{array}{l}
\beta\left(u_{n}\right)=0 \\
{\left[\left|\nabla u_{n}\right|^{2}+(1+\alpha(x)) u_{n}^{2}\right] d x \rightarrow m \text { (b) }}
\end{array}\right\} .
$$

Moreover $\left(A_{1}\right),(2.3)$ and (3.13)(b) imply $\lim _{n \rightarrow+\infty}\left\|u_{n}\right\|_{\mathbb{R}^{N}}^{2}=m$.

Then, by the uniqueness of the solution of (2.3), a sequence of points $\left(z_{n}\right)_{n}$ in $\mathbb{R}^{N}$ and a sequence of functions $\left(\varphi_{n}\right)_{n}$ in $H^{1}\left(\mathbb{R}^{N}\right)$ exist so that, up to a subsequence still denoted by $\left(u_{n}\right)_{n}$,

$$
\begin{gathered}
u_{n}(x)=w\left(x-z_{n}\right)+\varphi_{n}(x), \quad x \in \mathbb{R}^{N}, \\
\lim _{n \rightarrow+\infty} \varphi_{n}(x)=0 \quad \text { in } H^{1}\left(\mathbb{R}^{N}\right) \text { and in } L^{p}\left(\mathbb{R}^{N}\right)
\end{gathered}
$$

and, by the same arguments of Proposition 2.1, $\lim _{n \rightarrow+\infty}\left|z_{n}\right|=+\infty$.

On the other hand

$$
\lim _{n \rightarrow+\infty} \sup _{x \in \mathbb{R}^{N}}\left|\tilde{u}_{n}\left(x+z_{n}\right)-\widetilde{w}(x)\right|=0,
$$

and, as a consequence,

$$
\left|\beta\left(u_{n}(x)\right)-\beta\left(w\left(x-z_{n}\right)\right)\right| \rightarrow 0 \quad \text { as } n \rightarrow+\infty,
$$

that is

$$
\left|\beta\left(u_{n}(x)\right)-z_{n}\right| \rightarrow 0 \quad \text { as } n \rightarrow+\infty,
$$

contradicting (3.13)(a).

LEMMA 3.2. - Let $\Sigma, \psi_{\varepsilon, \rho}, \mathcal{B}_{0, \varepsilon}$ be as defined, respectively, in (3.8), (3.9), (3.4). Then for every $\rho>0$ there exists $\varepsilon_{\rho}>0$ such that for all $\varepsilon \in\left(0, \varepsilon_{\rho}\right)$

$$
\mathcal{B}_{0, \varepsilon} \leqslant \max _{\Sigma \times[0,1]} E_{\varepsilon}\left(\psi_{\varepsilon, \rho}[z, t]\right)
$$


Proof. - In view of (2.4), of the radial symmetry round 0 of $w(x)$ and of the fact that $\operatorname{dist}\left(\bar{\omega}_{\varepsilon}, 0\right) \rightarrow+\infty$ as $\varepsilon \rightarrow 0$, it is not difficult to verify that, for every fixed $\rho>0$,

$$
\lim _{\varepsilon \rightarrow 0} \max _{\Sigma}\left|\beta \circ \psi_{\varepsilon, \rho}[z, 0]-\rho z\right|=0 .
$$

Thus, for all $\varepsilon>0$ small enough, $\beta \circ \psi_{\varepsilon, \rho}(\Sigma \times\{0\})$ is homotopically equivalent in $\mathbb{R}^{N} \backslash\{0\}$ to $\rho \Sigma$ and, then, there exists $\left(\hat{z}_{\varepsilon}, \hat{t}_{\varepsilon}\right) \in \Sigma \times[0,1]$ such that $\beta \circ \psi_{\varepsilon, \rho}\left[\hat{z}_{\varepsilon}, \hat{t}_{\varepsilon}\right]=0$, hence

$$
\mathcal{B}_{0, \varepsilon} \leqslant E_{\varepsilon}\left(\psi_{\varepsilon, \rho}\left[\hat{z}_{\varepsilon}, \hat{t}_{\varepsilon}\right]\right) \leqslant \max _{\Sigma \times[0,1]} E_{\varepsilon}\left(\psi_{\varepsilon, \rho}[z, t]\right) .
$$

Proposition 3.3. - Let $\alpha$ satisfy $\left(A_{1}\right),\left(A_{2}\right)$ then there exist constants $\rho_{\alpha}>0$, $\mu_{\alpha}>0$ and $\varepsilon_{1}>0$, such that for all $\varepsilon \in\left(0, \varepsilon_{1}\right)$

$$
\begin{gathered}
\max _{\Sigma \times[0,1]} E_{\varepsilon}\left(\psi_{\varepsilon, \rho_{\alpha}}[z, t]\right)<\mu_{\alpha}<2^{1-2 / p} m, \\
\max _{\Sigma} E_{\varepsilon}\left(\psi_{\varepsilon, \rho_{\alpha}}[z, 0]\right)<\mathcal{B}_{0} .
\end{gathered}
$$

Proof. - The proof is carried out in three steps.

Step 1. There exists $\rho_{1}>0$ such that $\forall \rho>\rho_{1}$

$$
\max _{\Sigma \times[0,1]} \int_{\mathbb{R}^{N}}\left[\left|\nabla \psi_{\rho}[z, t]\right|^{2}+(1+\alpha(x))\left(\psi_{\rho}[z, t]\right)^{2}\right] d x:=\hat{\mu}_{\rho}<2^{1-2 / p} m .
$$

The argument is very similar to that of Lemma 3.5 in [8] so we only sketch it for the reader's convenience.

We define

$$
\begin{aligned}
N_{\rho}[z, t]= & \int_{\mathbb{R}^{N}}\left[|\nabla((1-t) w(x-\rho z)+t w(x-\rho \zeta))|^{2}\right. \\
& \left.+(1+\alpha(x))((1-t) w(x-\rho z)+t w(x-\rho \zeta))^{2}\right] d x, \\
D_{\rho}[z, t]= & |(1-t) w(x-\rho z)+t w(x-\rho \zeta)|_{p, \mathbb{R}^{N}}^{p} .
\end{aligned}
$$

To verify (3.17) we must prove that if $\rho$ is large enough

$$
\max _{\Sigma \times[0,1]} \frac{N_{\rho}[z, t]}{\left(D_{\rho}[z, t]\right)^{2 / p}}<2^{1-2 / p} m
$$

Taking into account that $-\Delta w+w=m w^{p-1}$ in $\mathbb{R}^{N}$ we obtain

$$
N_{\rho}[z, t]=\left[(1-t)^{2}+t^{2}\right] m+2 t(1-t) m \eta_{\rho}+2 t^{2} \theta_{\rho}+2(1-t)^{2} \delta_{\rho}
$$

where

$$
\eta_{\rho}=\int_{\mathbb{R}^{N}} w(x-\rho z)^{p-1} w(x-\rho \zeta) d x=\int_{\mathbb{R}^{N}} w(x-\rho z) w(x-\rho \zeta)^{p-1} d x,
$$




$$
\begin{aligned}
& \theta_{\rho}=\int_{\mathbb{R}^{N}} \alpha(x)|w(x-\rho \zeta)|^{2} d x, \\
& \delta_{\rho}=\int_{\mathbb{R}^{N}} \alpha(x)|w(x-\rho z)|^{2} d x .
\end{aligned}
$$

Using Lemma 2.2 of [1], (2.4) and condition $\left(A_{2}\right)$ we then deduce

$$
\begin{aligned}
& \lim _{\rho \rightarrow+\infty} \eta_{\rho}\left[2 \rho^{\frac{N-1}{2}} \mathrm{e}^{2 \rho}\right]=C_{1}>0, \\
& \lim _{\rho \rightarrow+\infty} \theta_{\rho}\left[\rho^{\frac{N-1}{2} \sigma} \mathrm{e}^{2 \rho}\right]=C_{2} \geqslant 0, \\
& \lim _{\rho \rightarrow+\infty} \delta_{\rho}\left[\rho^{\frac{N-1}{2} \sigma} \mathrm{e}^{2 \rho}\right]=C_{3} \geqslant 0,
\end{aligned}
$$

that allow to obtain

$$
N_{\rho}[z, t]=\left[(1-t)^{2}+t^{2}\right] m+2 t(1-t) m \eta_{\rho}+g(\rho)
$$

with $g(\rho)=o\left(\eta_{\rho}\right)$, because $\sigma \in(1,2]$.

On the other hand, using Lemma 2.7 of [8] we get

$$
D_{\rho}[z, t] \geqslant\left[(1-t)^{p}+t^{p}\right]+(p-1)\left[(1-t)^{p-1} t+t^{p-1}(1-t)\right] \eta_{\rho} .
$$

Hence

$$
\frac{N_{\rho}[z, t]}{\left(D_{\rho}[z, t]\right)^{2 / p}} \leqslant \frac{\left[(1-t)^{2}+t^{2}\right]}{\left[(1-t)^{p}+t^{p}\right]^{2 / p}} m+2 \gamma(t) m \eta_{\rho}+\mathrm{o}\left(\eta_{\rho}\right)
$$

where

$$
\gamma(t)=\frac{(1-t) t}{\left[(1-t)^{p}+t^{p}\right]^{2 / p}}\left\{1-\frac{p-1}{p} \frac{(1-t)^{2}+t^{2}}{(1-t)^{p}+t^{p}}\left[(1-t)^{p-2}+t^{p-2}\right]\right\} .
$$

Now $\gamma(1 / 2)<0$, so there exists a neighbourhood $I(1 / 2)$ such that $\gamma(t)<c<0$ $\forall t \in I(1 / 2)$ and

$$
\begin{aligned}
& \max \left\{\frac{N_{\rho}[z, t]}{\left(D_{\rho}[z, t]\right)^{2 / p}} \mid z \in \Sigma, t \in I\left(\frac{1}{2}\right)\right\} \\
& \leqslant 2^{1-2 / p} m+2 c m \eta_{\rho}+o\left(\eta_{\rho}\right)<2^{1-2 / p} m
\end{aligned}
$$

for $\rho$ large enough. Moreover the relation

$$
\begin{aligned}
& \lim _{\rho \rightarrow+\infty} \max \left\{\frac{N_{\rho}[z, t]}{\left(D_{\rho}[z, t]\right)^{2 / p}} \mid z \in \Sigma, t \in[0,1] \backslash I(1 / 2)\right\} \\
& =m \max \left\{\frac{\left[(1-t)^{2}+t^{2}\right]}{\left[(1-t)^{p}+t^{p}\right]^{2 / p}} \mid t \in[0,1] \backslash I(1 / 2)\right\}<2^{1-2 / p} m
\end{aligned}
$$

holds and together with (3.19) gives (3.18) as desired. 
Step 2. There exists $\hat{\rho} \geqslant \rho_{1}$ such that $\forall \rho \geqslant \hat{\rho}$

$$
\max _{\Sigma} \int_{\mathbb{R}^{N}}\left[\left|\nabla \psi_{\rho}[z, 0]\right|^{2}+(1+\alpha(x))\left(\psi_{\rho}[z, 0]\right)^{2}\right] d x<\mathcal{B}_{0}
$$

Since (3.12) holds and

$$
\begin{aligned}
\int_{\mathbb{R}^{N}} & {\left[\left|\nabla \psi_{\rho}[z, 0]\right|^{2}+(1+\alpha(x))\left(\psi_{\rho}[z, 0]\right)^{2}\right] d x } \\
& =\int_{\mathbb{R}^{N}}\left[|\nabla w(x-\rho z)|^{2}+(1+\alpha(x)) w(x-\rho z)^{2}\right] d x \\
& =m+\int_{\mathbb{R}^{N}} \alpha(x) w(x-\rho z)^{2} d x,
\end{aligned}
$$

to prove (3.20) we only need the relation

$$
\lim _{|\xi| \rightarrow+\infty} \int_{\mathbb{R}^{N}} \alpha(x) w(x-\xi)^{2} d x=0
$$

that follows, easily, arguing as in Proposition 2.1 to prove relation (2.10).

Step 3. Let $\rho_{\alpha} \geqslant \hat{\rho}$ and $\mu_{\alpha} \in\left(\hat{\mu}_{\rho_{\alpha}}, 2^{1-2 / p} m\right)$ be fixed, then there exists $\varepsilon_{1}>0$ such that (3.15) and (3.16) hold for all $\varepsilon \in\left(0, \varepsilon_{1}\right)$.

Because of the choice of $\rho_{\alpha}$, the inequalities (3.17) and (3.20) hold true when $\rho=\rho_{\alpha}$. Then in order to obtain (3.15) and (3.16) it is enough to observe that for all compact set $K \subset \Sigma \times[0,1]$

$$
\begin{aligned}
& \lim _{\varepsilon \rightarrow 0} \max _{(z, t) \in K} E_{\varepsilon}\left(\psi_{\varepsilon, \rho_{\alpha}}[z, t]\right) \\
& \quad=\max _{(z, t) \in K} \int_{\mathbb{R}^{N}}\left(\left|\nabla \psi_{\rho_{\alpha}}[z, t]\right|^{2}+(1+\alpha(x))\left(\psi_{\rho_{\alpha}}[z, t]\right)^{2}\right) d x .
\end{aligned}
$$

In fact, let $\varepsilon_{n}$ and $\left(z_{n}, t_{n}\right) \in K$ be such that $\lim _{n \rightarrow+\infty} \varepsilon_{n}=0$ and $\lim _{n \rightarrow+\infty}\left(z_{n}, t_{n}\right)=$ $\left(z_{0}, t_{0}\right) \in K$, then in view of (2.4) and of the fact that $\operatorname{dist}\left(\omega_{\varepsilon_{n}}, 0\right) \rightarrow+\infty$ it is not difficult to see that

$$
\lim _{n \rightarrow+\infty} \psi_{\varepsilon_{n}, \rho_{\alpha}}\left[z_{n}, t_{n}\right]=\psi_{\rho_{\alpha}}\left[z_{0}, t_{0}\right] \quad \text { in } H^{1}\left(\mathbb{R}^{N}\right)
$$

hence

$$
\lim _{n \rightarrow+\infty} E_{\varepsilon_{n}}\left(\psi_{\varepsilon_{n}, \rho_{\alpha}}\left[z_{n}, t_{n}\right]\right)=\int_{\mathbb{R}^{N}}\left(\left|\nabla \psi_{\rho_{\alpha}}\left[z_{0}, t_{0}\right]\right|^{2}+(1+\alpha(x))\left(\psi_{\rho_{\alpha}}\left[z_{0}, t_{0}\right]\right)^{2}\right) d x
$$

so (3.21) and the claim easily follow. 
Proposition 3.4. - Let $\mathcal{B}_{\bar{x}_{\varepsilon}}$ be as defined in (3.5). Let $\alpha$ satisfy $\left(A_{1}\right)$. Then there exists a constant $\mathcal{C}_{\bar{x}}>m$ such that the relation

$$
\mathcal{B}_{\bar{x}_{\varepsilon}} \geqslant \mathcal{C}_{\bar{x}}>m
$$

holds for all $\varepsilon>0$.

Proof. - To prove the claim, we argue by contradiction; so, we assume that a sequence $\left(\varepsilon_{n}\right)_{n}$ exists such that $\mathcal{B}_{\bar{x}_{\varepsilon_{n}}} \rightarrow m$, as $n \rightarrow+\infty$. We can also assume $\varepsilon_{n} \rightarrow 0$, as $n \rightarrow+\infty$, otherwise we get a contradiction at once, observing that $\varepsilon_{n} \geqslant \lambda>0$ for some $\lambda \in \mathbb{R}$ implies $\bar{x}_{\varepsilon_{n}} \in \widetilde{\omega}_{\lambda}:=\cup_{\varepsilon} \geqslant \lambda \omega_{\varepsilon}$ and

$$
\begin{gathered}
\mathcal{B}_{\bar{x}_{\varepsilon_{n}}} \geqslant \mathcal{C}_{\lambda}:=\inf \left\{\left.\int_{\mathbb{R}^{N}}\left(|\nabla u|^{2}+(1+\alpha(x)) u^{2}\right) d x\left|u \in H^{1}\left(\mathbb{R}^{N}\right),\right| u\right|_{p, \mathbb{R}^{N}}=1,\right. \\
\left.\beta(u) \in \widetilde{\omega}_{\lambda}\right\},
\end{gathered}
$$

and that, in view of the boundedness of $\widetilde{\omega}_{\lambda}$, arguing as in Proposition 3.1, it is not difficult to conclude $\mathcal{C}_{\lambda}>m$.

So a sequence of nonnegative functions $\left(u_{n}\right)_{n}, u_{n} \in H_{0}^{1}\left(\Omega_{\varepsilon_{n}}\right)$, must exist, such that $E_{\varepsilon_{n}}\left(u_{n}\right) \rightarrow m, \varepsilon_{n} \rightarrow 0$ as $n \rightarrow+\infty,\left|u_{n}\right|_{p, \Omega_{\varepsilon_{n}}}=1$ and $\beta\left(u_{n}\right)=\bar{x} / \varepsilon_{n}$. Hence there exist sequences $\left(z_{n}\right)_{n}$ in $\mathbb{R}^{N}$ and $\left(\varphi_{n}\right)_{n}$ in $H^{1}\left(\mathbb{R}^{N}\right)$ such that, up to a subsequence,

$$
u_{n}(x)=w\left(x-z_{n}\right)+\varphi_{n}(x) \quad \forall x \in \mathbb{R}^{N},
$$

and

$$
\lim _{n \rightarrow+\infty} \varphi_{n}(x)=0 \quad \text { strongly in } H^{1}\left(\mathbb{R}^{N}\right) \text { and in } L^{p}\left(\mathbb{R}^{N}\right) .
$$

So by the continuity of $\beta$, we infer

$$
\left|\frac{\bar{x}}{\varepsilon_{n}}-z_{n}\right|=\left|\beta\left(u_{n}\right)-z_{n}\right| \rightarrow 0 \quad \text { as } n \rightarrow+\infty
$$

from which the relation

$$
\lim _{n \rightarrow+\infty} \operatorname{dist}\left(\Omega_{\varepsilon_{n}}, z_{n}\right)=+\infty
$$

follows. Thus, for any $R>0$ and for $n$ large enough, $B\left(z_{n}, R\right) \cap \Omega_{\varepsilon_{n}}=\emptyset$ that implies

$$
\int_{B\left(z_{n}, R\right)}\left|u_{n}(x)\right| d x=0 .
$$

The above relation contradicts the relation

$$
\lim _{n \rightarrow+\infty} \int_{B\left(z_{n}, R\right)}\left|u_{n}(x)\right| d x=\int_{B(0, R)} w(x) d x>0
$$

that follows from the properties of $w$ and (3.23). 
Proposition 3.5. - Let $\alpha$ satisfy $\left(A_{1}\right)$. Let $\mathcal{A}_{\varepsilon}, \mathcal{B}_{0}, w_{\varepsilon, z}, \mathcal{C}_{\bar{x}}$ be as defined respectively in (3.7), (3.3), (3.10) and in Proposition 3.4. Let $R \in \mathbb{R}, R>0$ be chosen so that $\overline{B(0, R)} \subset \Omega$. Then there exists $\varepsilon_{2}>0$ such that

$$
m<\mathcal{A}_{\varepsilon} \leqslant \max _{|z|=R / 2 \varepsilon} E_{\varepsilon}\left(w_{\varepsilon, z}\right)<\min \left(\mathcal{B}_{0}, \mathcal{C}_{\bar{x}}\right)
$$

for all $\varepsilon \in\left(0, \varepsilon_{2}\right)$.

Proof. - Clearly, for every fixed $\varepsilon$, by the same arguments of Proposition $2.1, m<\mathcal{A}_{\varepsilon}$.

Let us, now, observe that, in view of (2.4), of the radial symmetry of $w$ and of the fact that $\operatorname{dist}\left(\partial B(0, R / 2 \varepsilon), \bar{\omega}_{\varepsilon}\right) \rightarrow+\infty$ as $\varepsilon \rightarrow 0$, we have

$$
\lim _{\varepsilon \rightarrow 0} \max _{|z|=R / 2 \varepsilon}\left\|w_{\varepsilon, z}(x)-w(x-z)\right\|_{\mathbb{R}^{N}}=0
$$

and

$$
\lim _{\varepsilon \rightarrow 0} \max _{|z|=R / 2 \varepsilon}\left|\beta\left(w_{\varepsilon, z}\right)-z\right|=0 .
$$

(3.25) implies $\lim _{\varepsilon \rightarrow 0} \max _{|z|=R / 2 \varepsilon} E_{\varepsilon}\left(w_{\varepsilon, z}\right)=m$ and this relation, with (3.12) and (3.22), gives the third inequality for small $\varepsilon$.

As a consequence of (3.26), for small $\varepsilon$, the map

$$
z \rightarrow \beta\left(w_{\varepsilon, z}\right)
$$

is homotopic to the identity map $i$ on $\partial B(0, R / 2 \varepsilon)$ by the homotopy

$$
\mathcal{K}(\theta, z)=\theta \beta\left(w_{\varepsilon, z}\right)+(1-\theta) z, \quad 0 \leqslant \theta \leqslant 1,
$$

and $\mathcal{K}(\theta, z) \notin\left\{0, \bar{x}_{\varepsilon}\right\}, \forall \theta \in[0,1] \forall z \in \partial B(0, R / 2 \varepsilon)$.

Then there exists $\tilde{z} \in \partial B(0, R / 2 \varepsilon)$ such that $\beta\left(w_{\varepsilon, \tilde{z}}\right) \in L_{\varepsilon}$, hence the relation

$$
\mathcal{A}_{\varepsilon} \leqslant E_{\varepsilon}\left(w_{\varepsilon, \tilde{z}}\right) \leqslant \max _{|z|=R / 2 \varepsilon} E_{\varepsilon}\left(w_{\varepsilon, z}\right)
$$

gives the second inequality.

Proposition 3.6. - Let $\alpha$ satisfy $\left(A_{1}\right)$. Let $\mathcal{B}_{0, \beta_{\varepsilon}}$ be as defined in (3.6). Let $\mu$ be a constant such that $\mu \in\left(m, 2^{1-2 / p} m\right)$ then there exists $\varepsilon_{\mu}>0$ such that

$$
\mathcal{B}_{0, \beta_{\varepsilon}}>\mu
$$

for all $\varepsilon \in\left(0, \varepsilon_{\mu}\right)$.

Proof. - The claim follows from the asymptotic estimate

$$
\lim _{\varepsilon \rightarrow 0} \mathcal{B}_{0, \beta_{\varepsilon}}=2^{1-2 / p} m
$$

that can be obtained arguing exactly as in Lemma 3.3 and Remark 3.4 of [15]. 
Lemma 3.7. - Let $\Sigma, \psi_{\varepsilon, \rho}, \mathcal{B}_{0, \beta_{\varepsilon}}$ be as defined respectively in (3.8), (3.9), (3.6). Then for every $\varepsilon>0$ there exists $\hat{\rho}_{\varepsilon}>0$ such that for all $\rho>\hat{\rho}_{\varepsilon}$

$$
\mathcal{B}_{0, \beta_{\varepsilon}} \leqslant \max _{\Sigma \times[0,1]} E_{\varepsilon}\left(\psi_{\varepsilon, \rho}[z, t]\right) .
$$

Proof. - In view of (2.4), of the radial symmetry of $w$ and by the definition (3.2) of $\beta_{\varepsilon}$, it is not difficult to verify that, for every fixed $\varepsilon>0$,

$$
\lim _{\rho \rightarrow+\infty} \max _{z \in \Sigma}\left|\beta_{\varepsilon} \circ \psi_{\varepsilon, \rho}[z, 0]-\chi\left(\rho z-\bar{x}_{\varepsilon}\right)\right|=0 .
$$

Hence, for all $\rho$ large enough, the set $\beta_{\varepsilon} \circ \psi_{\varepsilon, \rho}(\Sigma \times\{0\})$ is homotopically equivalent in $\mathbb{R}^{N} \backslash\{0\}$ to $\rho \Sigma$ and, then, there exists $\left(\bar{z}_{\rho}, \bar{t}_{\rho}\right) \in \Sigma \times[0,1]$ such that $\beta_{\varepsilon} \circ \psi_{\varepsilon, \rho}\left(\bar{z}_{\rho}, \bar{t}_{\rho}\right)=0$, thus

$$
\mathcal{B}_{0, \beta_{\varepsilon}} \leqslant E_{\varepsilon}\left(\psi_{\varepsilon, \rho}\left(\bar{z}_{\rho}, \bar{t}_{\rho}\right)\right) \leqslant \max _{\Sigma \times[0,1]} E_{\varepsilon}\left(\psi_{\varepsilon, \rho}[z, t]\right) .
$$

Proposition 3.8. - Let $\alpha$ satisfy $\left(A_{1}\right)$ and let $\mu$ be so that $\mu \in\left(m, 2^{1-2 / p} m\right)$. For every $\varepsilon>0$ there exists $\bar{\rho}_{\varepsilon, \mu}>0$ such that for all $\rho \geqslant \bar{\rho}_{\varepsilon, \mu}$

$$
\begin{gathered}
\max _{\Sigma \times[0,1]} E_{\varepsilon}\left(\psi_{\varepsilon, \rho}[z, t]\right)<2^{1-2 / p} m, \\
\max _{\Sigma} E_{\varepsilon}\left(\psi_{\varepsilon, \rho}[z, 0]\right)<\mu .
\end{gathered}
$$

Proof. - The proof is carried out in three steps.

Step 1. For every $\varepsilon>0$ there exists $\bar{\rho}_{\varepsilon, 1}>0$ such that for all $\rho>\bar{\rho}_{\varepsilon, 1}$

$$
\max _{\Sigma \times[0,1]} \int_{\Omega_{\varepsilon}}\left[\left|\nabla \psi_{\varepsilon, \rho}[z, t]\right|^{2}+\left(\psi_{\varepsilon, \rho}[z, t]\right)^{2}\right] d x \leqslant 2^{1-2 / p} m .
$$

The proof of this step is just Lemma 3.5 in [8].

Step 2. For every $\varepsilon>0$ there exists $\bar{\rho}_{\varepsilon, 2}>\bar{\rho}_{\varepsilon, 1}$ such that

$$
\max _{\Sigma} \int_{\Omega_{\varepsilon}}\left[\left|\nabla \psi_{\varepsilon, \rho}[z, 0]\right|^{2}+\left(\psi_{\varepsilon, \rho}[z, 0]\right)^{2}\right] d x \leqslant \mu
$$

holds for all $\rho>\bar{\rho}_{\varepsilon, 2}$.

By (2.4), the shape of $w$ and the choice of $\phi_{\varepsilon}$ we have

$$
\lim _{|z| \rightarrow+\infty}\left\|\phi_{\varepsilon}(x) w(x-z)-w(x-z)\right\|_{\mathbb{R}^{N}}=0
$$

from which

$$
\lim _{\rho \rightarrow \infty} \max _{\Sigma}\left[\left\|\psi_{\varepsilon, \rho}[z, 0]\right\|_{\mathbb{R}^{N}}^{2}-\|w(x-\rho z)\|_{\mathbb{R}^{N}}^{2}\right]=0
$$


that implies

$$
\lim _{\rho \rightarrow+\infty} \max _{\Sigma} \int_{\Omega_{\varepsilon}}\left[\left|\nabla \psi_{\varepsilon, \rho}[z, 0]\right|^{2}+\left(\psi_{\varepsilon, \rho}[z, 0]\right)^{2}\right] d x=m .
$$

Step 3. For every $\varepsilon>0$ there exists $\bar{\rho}_{\varepsilon}>\bar{\rho}_{\varepsilon, 2}$ such that (3.30) and (3.31) hold for all $\rho>\bar{\rho}_{\varepsilon}$.

Taking into account that $\left|\phi_{\varepsilon}(x)[(1-t) w(x-\rho z)+t w(x-\rho \zeta)]\right|_{p, \Omega_{\varepsilon}} \geqslant c>0$, arguing as in Proposition 2.1 to prove (2.10) it is not difficult to see that

$$
\lim _{\rho \rightarrow+\infty} \max _{\Sigma \times[0,1]} \int_{\Omega_{\varepsilon}} \alpha(x)\left(\psi_{\varepsilon, \rho}[z, t](x)\right)^{2} d x=0 .
$$

Hence

$$
\lim _{\rho \rightarrow+\infty} \max _{\Sigma \times[0,1]}\left[E_{\varepsilon}\left(\psi_{\varepsilon, \rho}[z, t]\right)-\int_{\Omega_{\varepsilon}}\left[\left|\nabla \psi_{\varepsilon, \rho}[z, t]\right|^{2}+\left(\psi_{\varepsilon, \rho}[z, t]\right)^{2}\right] d x\right]=0
$$

that, with (3.32) and (3.33), gives (3.30) and (3.31).

\section{Proof of Theorem 1.1}

To prove the theorem we show that, for small $\varepsilon, E_{\varepsilon}$ has on $M_{\varepsilon}$ three distinct critical values, lying in the energy range $\left(m, 2^{1-2 / p} m\right)$, to which there correspond at least three distinct solutions of $\left(P_{\varepsilon}\right)$, positive by Lemma 2.3.

In what follows $\rho_{\alpha}, \mu_{\alpha}$ are the constants whose existence is stated in Proposition 3.3, moreover we choose $\bar{\varepsilon}=\min \left(\varepsilon_{\rho_{\alpha}}, \varepsilon_{1}, \varepsilon_{2}, \varepsilon_{\mu_{\alpha}}\right)$ where $\varepsilon_{1}, \varepsilon_{2}$ are, respectively, the numbers found in Propositions 3.3 and 3.5 and $\varepsilon_{\rho_{\alpha}}, \varepsilon_{\mu_{\alpha}}$ are as stated in Lemma 3.2 and Proposition 3.6.

We remark that, by the results of Section 3 , for all $\varepsilon \in(0, \bar{\varepsilon})$ the following inequalities hold

$$
\begin{aligned}
m & <\mathcal{A}_{\varepsilon} \leqslant \max _{|z|=R / 2 \varepsilon} E_{\varepsilon}\left(w_{\varepsilon, z}\right)<\min \left(\mathcal{B}_{0}, \mathcal{C}_{\bar{x}}\right), \\
\max _{\Sigma} E_{\varepsilon}\left(\psi_{\varepsilon, \rho_{\alpha}}[z, 0]\right) & <\mathcal{B}_{0} \leqslant \mathcal{B}_{0, \varepsilon} \leqslant \max _{\Sigma \times[0,1]} E_{\varepsilon}\left(\psi_{\varepsilon, \rho_{\alpha}}[z, t]\right) \\
& <\mu_{\alpha}<\mathcal{B}_{0, \beta_{\varepsilon}}<2^{1-2 / p} m .
\end{aligned}
$$

and, fixed $\varepsilon \in(0, \bar{\varepsilon})$, for all $\rho>\max \left(\hat{\rho}_{\varepsilon}, \bar{\rho}_{\varepsilon, \mu_{\alpha}}, \rho_{\alpha}\right)\left(\hat{\rho}_{\alpha}\right.$ and $\bar{\rho}_{\varepsilon, \mu_{\alpha}}$ being the numbers whose existence is stated in Lemma 3.7 and Proposition 3.8, respectively)

$$
\max _{\Sigma} E_{\varepsilon}\left(\psi_{\varepsilon, \rho}[z, 0]\right)<\mu_{\alpha}<\mathcal{B}_{0, \beta_{\varepsilon}} \leqslant \max _{\Sigma \times[0,1]} E_{\varepsilon}\left(\psi_{\varepsilon, \rho}[z, t]\right)<2^{1-2 / p} m .
$$

We consider a fixed $\varepsilon \in(0, \bar{\varepsilon})$ and we carry out the proof in three steps: first we prove, in Step 1, the existence of a critical value $c_{1, \varepsilon}$ satisfying

$$
\mathcal{A}_{\varepsilon} \leqslant c_{1, \varepsilon} \leqslant \max _{|z|=R / 2 \varepsilon} E_{\varepsilon}\left(w_{\varepsilon, z}\right)
$$


then, in Step 2, we show that another critical level $c_{2, \varepsilon}$ exists so that

$$
\mathcal{B}_{0, \varepsilon} \leqslant c_{2, \varepsilon} \leqslant \max _{\Sigma \times[0,1]} E_{\varepsilon}\left(\psi_{\varepsilon, \rho_{\alpha}}[z, t]\right),
$$

finally, in Step 3, we state the existence of a third critical level $c_{3, \varepsilon} \geqslant \mathcal{B}_{0, \beta_{\varepsilon}}$.

The above levels are distinct because, by (4.1), (4.2),

$$
m<c_{1, \varepsilon}<\mathcal{B}_{0} \leqslant c_{2, \varepsilon}<\mu_{\alpha}<c_{3, \varepsilon}<2^{1-2 / p} m .
$$

Moreover, since, by (3.25), $\lim _{\varepsilon \rightarrow 0} \max _{|z|=R / 2 \varepsilon} E_{\varepsilon}\left(w_{\varepsilon, z}\right)=m$, and, by Proposition 3.6, the asymptotic estimate $\lim _{\varepsilon \rightarrow 0} \mathcal{B}_{0, \beta_{\varepsilon}}=2^{1-2 / p} m$ holds, using again (4.1), we deduce

$$
\lim _{\varepsilon \rightarrow 0} c_{1, \varepsilon}=m, \quad \lim _{\varepsilon \rightarrow 0} c_{2, \varepsilon} \in\left[\mathcal{B}_{0}, \mu_{\alpha}\right] \subset\left(m, 2^{1-2 / p} m\right), \quad \lim _{\varepsilon \rightarrow 0} c_{3, \varepsilon}=2^{1-2 / p} m,
$$

that, with (2.1), imply (1.2)-(1.4).

In what follows, for a given $\gamma \in \mathbb{R}$, we set $E_{\varepsilon}^{\gamma}=\left\{u \in M_{\varepsilon} \mid E_{\varepsilon}(u) \leqslant \gamma\right\}$. that

Step 1 . Let us denote by $\mathcal{S}_{R, \varepsilon}=\max _{|z|=R / 2 \varepsilon} E_{\varepsilon}\left(w_{\varepsilon, z}\right)$. We assume, by contradiction,

$$
\left\{u \in M_{\varepsilon} \mid \mathcal{A}_{\varepsilon} \leqslant E_{\varepsilon}(u) \leqslant \mathcal{S}_{R, \varepsilon}, \nabla E_{\varepsilon \mid M_{\varepsilon}}(u)=0\right\}=\emptyset .
$$

Since the pair $\left(E_{\varepsilon}, M_{\varepsilon}\right)$ satisfies the Palais-Smale condition, using a well known deformation lemma (see f.i. [17]), we find a positive number $\delta_{1}>0$ and a continuous $\operatorname{map} \eta:[0,1] \times E_{\varepsilon}^{\mathcal{S}_{R, \varepsilon}} \rightarrow E_{\varepsilon}^{\mathcal{S}_{R, \varepsilon}}$ such that

$$
\begin{aligned}
& \eta(0, u)=u, \quad \forall u \in E_{\varepsilon}^{\mathcal{S}_{R, \varepsilon}}, \\
& \eta\left(1, E_{\varepsilon}^{\mathcal{S}_{R, \varepsilon}}\right) \subseteq E_{\varepsilon}^{\mathcal{A}_{\varepsilon}-\delta_{1}} .
\end{aligned}
$$

Then we define $\forall \theta \in[0,1]$ and $\forall z \in \partial B(0, R / 2 \varepsilon)$ the continuous map

$$
\mathcal{G}(\theta, z)= \begin{cases}\mathcal{K}(2 \theta, z) & 0 \leqslant \theta \leqslant 1 / 2, \\ \beta\left(\eta\left(2 \theta-1, w_{\varepsilon, z}\right)\right) & 1 / 2 \leqslant \theta \leqslant 1,\end{cases}
$$

$\mathcal{K}$ being the map defined in (3.27). By the definition of $\mathcal{K}, \mathcal{G}(\theta, z) \notin\left\{0, \bar{x}_{\varepsilon}\right\} \forall \theta \in$ $[0,1 / 2] \forall z \in \partial B(0, R / 2 \varepsilon)$, moreover, by the relations (4.1) $\mathcal{S}_{R, \varepsilon}<\min \left(\mathcal{B}_{0}, \mathcal{C}_{\bar{x}}\right) \leqslant$ $\min \left(\mathcal{B}_{0, \varepsilon}, \mathcal{B}_{\bar{x}_{\varepsilon}}\right), \mathcal{G}(\theta, z) \notin\left\{0, \bar{x}_{\varepsilon}\right\} \forall \theta \in[1 / 2,1], \forall z \in \partial B(0, R / 2 \varepsilon)$. Hence, taking into account that $\mathcal{K}(0, z)=z \forall z \in \partial B(0, R / 2 \varepsilon)$, we deduce the existence of $\hat{z} \in \partial B(0, R / 2 \varepsilon)$ such that

$$
\mathcal{G}(1, \hat{z})=\beta \circ \eta\left(1, w_{\varepsilon, \hat{z}}\right) \in L_{\varepsilon} .
$$

On the other hand by (4.3) and (3.7)

$$
\mathcal{G}(1, \partial B(0, R / 2 \varepsilon)) \cap L_{\varepsilon}=\emptyset,
$$

that contradicts (4.4).

Step 2. Set $\mathcal{Q}_{\rho_{\alpha}, \varepsilon}=\max _{\Sigma \times[0,1]} E_{\varepsilon}\left(\psi_{\varepsilon, \rho_{\alpha}}[z, t]\right)$. We assume, by contradiction, that

$$
\left\{u \in M_{\varepsilon} \mid \mathcal{B}_{0, \varepsilon} \leqslant E_{\varepsilon}(u) \leqslant \mathcal{Q}_{\rho_{\alpha}, \varepsilon}, \nabla E_{\varepsilon \mid M_{\varepsilon}}(u)=0\right\}=\emptyset,
$$


then, arguing as in the previous step, we find a number $\delta_{2}>0$ and a continuous function $\sigma: E_{\varepsilon}^{\mathcal{Q}_{p_{\alpha}, \varepsilon}} \rightarrow E_{\varepsilon}^{\mathcal{B}_{0, \varepsilon}-\delta_{2}}$ such that

$$
\sigma(u)=u \quad \forall u \in E_{\varepsilon}^{\mathcal{B}_{0, \varepsilon}-\delta_{2}},
$$

furthermore, by (3.12) and (3.16), $\delta_{2}$ can be chosen in such a way that

$$
\max _{\Sigma} E_{\varepsilon}\left(\psi_{\varepsilon, \rho_{\alpha}}[z, 0]\right)<\mathcal{B}_{0, \varepsilon}-\delta_{2}
$$

Setting

$$
\widetilde{\Sigma}=\frac{\Sigma \times[0,1]}{\sim}
$$

where $\sim$ identifies the points $(z, 1)$, we define a map $\mathcal{H}$ on $\widetilde{\Sigma}$ by

$$
\mathcal{H}[z, t]=\beta\left(\sigma\left(\psi_{\varepsilon, \rho_{\alpha}}[z, t]\right)\right) .
$$

Since $\varepsilon<\varepsilon_{\rho_{\alpha}}$, by Lemma 3.2, (4.5) and (4.6), $\mathcal{H}$ maps $\partial \widetilde{\Sigma}$ in a set homotopically equivalent to $\rho_{\alpha} \Sigma$ (and then to $\Sigma$ ) in $\mathbb{R}^{N} \backslash\{0\}$. Moreover $\mathcal{H}$ is continuous, so a point $(\tilde{z}, \tilde{t}) \in \widetilde{\Sigma}$ must exist, for which

$$
0=\mathcal{H}(\tilde{z}, \tilde{t})=\beta\left(\sigma\left(\psi_{\varepsilon, \rho_{\alpha}}[\tilde{z}, \tilde{t}]\right)\right) .
$$

This is impossible because $\sigma(\widetilde{\Sigma}) \subset \sigma\left(E_{\varepsilon}^{\mathcal{Q}_{\rho_{\alpha}, \varepsilon}}\right) \subset E_{\varepsilon}^{\mathcal{B}_{0, \varepsilon}-\delta_{2}}$ and by (3.4), so we are in contradiction.

Step 3. Considering a fixed $\rho>\max \left(\hat{\rho}_{\varepsilon}, \bar{\rho}_{\varepsilon, \mu_{\alpha}}, \rho_{\alpha}\right)$, taking into account (4.2) and using the same argument displayed in Step 2, we deduce, as desired, that

$$
\left\{u \in M_{\varepsilon} \mid \mathcal{B}_{0, \beta_{\varepsilon}} \leqslant E_{\varepsilon}(u) \leqslant \max _{\Sigma \times[0,1]} E_{\varepsilon}\left(\psi_{\varepsilon, \rho}[z, t]\right), \nabla E_{\varepsilon \mid M_{\varepsilon}}(u)=0\right\} \neq \emptyset .
$$

\section{REFERENCES}

[1] A. Bahri, P.L. Lions, On the existence of a positive solution of semilinear elliptic equations in unbounded domains, Ann. Inst. H. Poincaré Anal. Non Linéaire 14 (3) (1997) 365-413.

[2] A. Bahri, Y.Y. Li, On a min-max procedure for the existence of a positive solution for certain scalar field equations in $\mathbb{R}^{N}$, Rev. Mat. Iberoamericana 6 (1-2) (1990) 1-15.

[3] V. Benci, G. Cerami, Positive solutions of some nonlinear elliptic problems in exterior domains, Arch. Rational Mech. Anal. 99 (1987) 283-300.

[4] V. Benci, G. Cerami, Existence of positive solutions of the equation $-\Delta u+a(x) u=$ $u^{(N+2) /(N-2)}$ in $\mathbb{R}^{N}$, J. Funct. Anal. 88 (1) (1990) 90-117.

[5] H. Berestycki, P.L. Lions, Nonlinear scalar fields equations - I. Existence of a ground-state, Arch. Rational Mech. Anal. 82 (1983) 313-346.

[6] G. Cerami, C. Maniscalco, Multiple positive solutions for a singularly perturbed Dirichlet problem in "geometrically trivial" domains, Topol. Methods Nonlin. Anal. 19 (1) (2002) 63-76. 
[7] G. Cerami, D. Passaseo, Existence and multiplicity of positive solutions for nonlinear elliptic problems in exterior domains with "rich" topology, Nonlinear Analysis TMA 18 (2) (1992) 109-119.

[8] G. Cerami, D. Passaseo, Existence and multiplicity results for semilinear elliptic Dirichlet problems in exterior domains, Nonlinear Analysis TMA 24 (11) (1995) 1533-1547.

[9] G. Cerami, D. Passaseo, Effect of concentrating potentials in some singularly perturbed problems, Calculus of Variations and PDE, to appear.

[10] B. Gidas, W.M. Ni, L. Nirenberg, Symmetry of positive solutions of nonlinear elliptic equations in $\mathbb{R}^{N}$, in: Mathematical Analysis and Applications, Part A, in: Advances in Mathematics Supplementary Studies, Vol. 7-A, Academic Press, 1981, pp. 369-402.

[11] M. Grossi, D. Passaseo, Nonlinear elliptic Dirichlet problems in exterior domains: the role of geometry and topology of the domain, Comm. Appl. Nonlinear Anal. 2 (2) (1995) 1-31.

[12] M.K. Kwong, Uniqueness of positive solutions of $\Delta u-u+u^{p}=0$, Arch. Rational Mech. Anal. 105 (1989) 243-266.

[13] R. Molle, M. Musso, D. Passaseo, Positive solutions for a class of nonlinear elliptic problems in $\mathbb{R}^{N}$, Proc. Roy. Soc. Edinburgh Sect. A 130 (1) (2000) 141-166.

[14] R. Molle, D. Passaseo, On the behaviour of the solutions for a class of nonlinear elliptic problems in exterior domains, Discrete Contin. Dynam. Systems 4 (3) (1998) 445-454.

[15] R. Molle, D. Passaseo, Multiple solutions of nonlinear elliptic Dirichlet problems in exterior domains, Nonlinear Anal. Ser. A: Theory Methods 39 (4) (2000) 447-462.

[16] W.A. Strauss, Existence of solitary waves in higher dimensions, Comm. Math. Phys. 55 (1977) 149-162.

[17] M. Struwe, Variational Methods - Applications to Nonlinear Partial Differential Equations and Hamiltonian Systems, Springer-Verlag, Berlin, 1990. 